\title{
La Evaluación Educativa en el Currículo LOMCE en Primaria: Análisis de los Currículos Autonómicos en Educación Física
}

\author{
The Educational Evaluation in LOMCE Curriculum in Primary: \\ Analysis of the Autonomic Curricula in Physical Education
}

\author{
Fernando Manuel Otero-Saborido * \\ Francisco Javier Vázquez-Ramos \\ Universidad Pablo de Olavide, España
}

\begin{abstract}
La evaluación es uno de los aspectos que determina todo el proceso de enseñanza aprendizaje. Debido a sus connotaciones éticas es un proceso que debe ser objeto de análisis. Por ello, el objeto general de este trabajo fue realizar un análisis de los aspectos evaluativos en Educación Física comparando los 17 currículos autonómicos que emanan del reglamento estatal preceptuado en el Real Decreto 126/2014. Para ello se realizó un análisis documental como instrumento de obtención informativa de los 17 reglamentos autonómicos que desarrollan los aspectos evaluativos en Educación Física. Tras la circulación por un grupo de expertos resultaron un total de 12 variables a comparar y analizar. Los resultados del análisis muestran la tendencia a la eliminación a los objetivos de área, el aumento preponderante de criterios $(M=61,53)$ y, sobre todo, de estándares de aprendizaje $(M=200,24)$ que la mayoría de currículos secuencia por niveles educativos. Así mismo, cabe resaltar la casi inexistencia en la mayoría de Comunidades Autónomas de orientaciones para una evaluación más educativa en el área de Educación Física.
\end{abstract}

Palabras clave: Evaluación formativa; Estándares de aprendizaje; Criterios de evaluación; Medición; Educación primaria.

\begin{abstract}
Asssessment is one of the aspects that determines the entire teaching-learning process. Due to its ethical connotations it is a process that must be analyzed. Therefore, the general purpose of this work was to perform an analysis of the evaluation aspects in Physical Education comparing the 17 regional curricula that emanate from the state regulation prescribed in Royal Decree 126/2014. To this end, a documentary analysis was carried out as an instrument to obtain information on the 17 regional regulations that develop the evaluation aspects in Physical Education. After the circulation by a group of experts, a total of 12 variables were analyzed and analyzed. The results of the analysis show the tendency to elimination to the area objectives, the preponderant increase of criteria $(M=61.53)$ and, above all, of learning standards $(\mathrm{M}=200.24)$ than the majority of curriculums sequencing by educational levels. Likewise, it is worth highlighting the almost nonexistence in most Autonomous Communities of guidelines for a more educational evaluation in the area of Physical Education.
\end{abstract}

Keywords: Formative assessment; Learning standards; Evaluation criteria; Measurement; Primary education.

*Contacto: fmotero@upo.es

issn: 1989-0397

www.rinace.net/riee/

https://revistas.uam.es/riee
Recibido: $\quad 15$ de enero de 2019

$1^{\text {a }}$ Evaluación: 07 de febrero de 2019

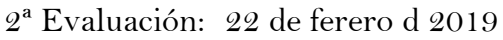

Aceptado: $\quad 26$ de febrero de 2019 


\section{Introducción}

La evaluación es uno de los aspectos más controvertidos en las instituciones educativas debido a las connotaciones éticas e ideológicas que emanan como fenómeno dentro del proceso de enseñanza aprendizaje (Santos-Guerra, 2006). La prueba de que la evaluación transciende lo educativo hasta llegar a ámbitos macropolíticos y económicos es la existencia de organismos internacionales (OCDE) destinados a medir los rendimientos escolares (Biesta, 2014) y estandarizar pruebas de medición (PISA, TIMMS, PIRLS...) cuyos resultados orienten las políticas educativas públicas. El auge de la cultura de la medición ha reducido las acepciones y dimensiones educativas y sociales de la evaluación al pretender comprender y evaluar el sistema educativo sólo a través de los resultados sin mirar el proceso. Una verdadera cultura de evaluación educativa "implica conocer cómo se desarrollan los procesos de formación para mejorar los aspectos más necesarios y potenciar los positivos" (Díez-Gutiérrez, 2018, p. 57). La evitación del proceso — de lo que ocurre durante - para centrarse exclusivamente en los resultados y la ignorancia de las condiciones del aprendizaje a la hora calificar y/o seleccionar discentes o instituciones educativas es providencialmente conceptualizada por Alcaraz (2015) en su reflexión sobre el concepto de evaluación educativa: "Decimos que evaluamos para comprobar si el alumnado está aprendiendo o no y olvidamos que la principal función de la evaluación no es tanto comprobar el aprendizaje como asegurar las condiciones para que se produzca dicho aprendizaje"(p.210). En este sentido, no es casual la preponderancia de la función selectiva (excluyente) de la evaluación en detrimento de la función formadora (inclusiva) (Pozo, 2016) y, sobre todo, que esa función selectiva recaiga sólo en alumnado y docentes y no valore recursos, otros agentes y, sobre todo, las políticas educativas del que ejerce el poder de evaluar (Santos-Guerra, 2006, p. 42).

Además, desde un punto de vista epistemológico, existe un sesgo de hondura a la hora de afrontar la evaluación educativa. En las ciencias de la educación hemos importado tradicionalmente los métodos empíricos-analíticos de las ciencias naturales que son insuficientes e inadecuados por sí solos en nuestro objeto de trabajo: la interacción social entre los seres humanos en un centro educativo. Sin embargo, las ciencias de la educación han construido su propio marco de referencia, ya que poseen instrumentos y estrategias para comprender y transformar los enconados modelos evaluativos del pasado que sobreviven en el presente. Por el contrario, el enfoque científico-natural busca explicaciones con perfectas relaciones causa-efecto que difícilmente se manifiestan en los entornos escolares, pretenden formular leyes infalibles, en definitiva, generalizar, pero pocos o ningún contexto educativo es generalizable dada su singularidad (López-Pastor, 1999). En cambio, los enfoques interpretativos de las ciencias de la educación intentan comprender y profundizar en cada contexto. En esa línea es certera la contribución de Hopkins (1989) cuándo señala que los métodos cuantitativos de investigación (y por ende de evaluación) nos pueden ayudar a trazar una hipótesis de trabajo en el aula, nos colocan tras una pista, pero cada investigación sobre educación debe ser comprobada en las condiciones particulares sin que se pueda generalizar ya que en cada lugar se aprende de diferente forma. Así lo señala Crobanch (1975) para referirse a los métodos positivistas: "Cuando sopesamos convenientemente las condiciones particulares, cualquier generalización es un hipótesis de trabajo, no una conclusión” (p.125). 
La exportación a la EF (Educación Física, a partir de ahora) de los dos modelos señalados anteriormente tiene una descripción homogénea. Un primer modelo orientado hacia el rendimiento físico que tiene como objetivo medir la eficacia del rendimiento físico del alumno a través de test y pruebas estandarizadas. Esta concepción no contempla el valor formativo de la evaluación. Su intención la resume la pregunta de López-Pastor (2004) “¿Por qué decimos evaluación cuándo en realidad queremos decir calificación?” En oposición, el modelo orientado hacia la participación del alumno sí tiene un alto valor formativo. No valora tan sólo el aspecto motriz, sino que contempla las dimensiones afectiva, social y cognitiva a través de la implicación del alumnado en la evaluación (LópezPastor, 1999). Existe ya una línea consolidada de evidencias científicas que han demostrado los beneficios de implicar al alumnado al utilizando situaciones de autoevaluación y de evaluación por pares o coevaluación en Educación Física (Lamb, Lane, y Aldous 2013; López-Pastor et al. 2007; Ní Chróinín y Cosgrave, 2013; Pérez, Heras, y Hernán 2008; Vera, González, y Moreno 2009).

No obstante, las revisiones de diferentes trabajos sobre prácticas de evaluación en diferentes contextos y momentos indican que si bien el paradigma teórico indica la prevalencia de una evaluación formativa, en las prácticas evaluativas de los docentes de EF siguen predominando los modelos de calificación y con escasa participación del alumnado (Lorente y Kirk, 2014; Otero, 2014; Otero, Calvo y González, 2014). Esa incoherencia entre paradigmas teóricos de evaluación y lo que ocurre en las prácticas educativas del aula es potenciada cuando el profesorado tiene como referencia normativa currículos por objetivos que cierran la evaluación y la reducen a una comprobación técnica de éxito y logro. Estos currículos — que serán objeto de análisis documental a lo largo de este trabajo- destinados a la "estandarización" de la evaluación tienen como principal ventaja pudiera resaltarse que facilitan la medición, que no evaluación, del alumnado. Como desventaja reducen el "conocimiento a conductas, no sirven para el desarrollo del profesor y, en definitiva, pueden alcanzarse los objetivos propuestos sin educar a los alumnos" (Stenhouse, 1987, p.14). Más concretamente, el Real Decreto 126/2014, de 28 de febrero, por el que se establece el currículo básico de la Educación Primaria, tipifica dos elementos curriculares que entroncan la evaluación: criterios de evaluación (CE, a partir de ahora) y estándares de aprendizajes evaluables (EA, partir de ahora). Si bien los CE entendidos por la normativa citada como "el referente específico para evaluar el aprendizaje del alumnado" no son elementos nuevos sino que ya existían en los desarrollos reglamentarios previos a la LOMCE; no es el caso de los EA cuyo Real Decreto los entiende como "especificaciones de los criterios de evaluación que permiten definir los resultados de aprendizaje, y que concretan lo que el alumno debe saber, comprender y saber hacer en cada asignatura; deben ser observables, medibles y evaluables y permitir graduar el rendimiento o logro alcanzado. Su diseño debe contribuir y facilitar el diseño de pruebas estandarizadas y comparables". En este caso, la inclusión de los EA que desarrollan casi todas las Comunidades Autonómicas en sus currículos son el elemento distintivo y la nueva seña de identidad curricular evaluativa que coloca a la evaluación como "medible", "estandarizada", "observable" y con carácter de "rendimiento" sin que se pueda encontrar en el Reglamento curricular del que beben los 17 currículos autonómicos ninguna referencia al carácter formativo ni a la participación del alumnado en los procesos de evaluación. Si bien en la línea del análisis curricular se encontraron referencias para la asignatura de EF, ninguno de ellos se ocupa de forma específica de la evaluación en todas 
las Comunidades Autónomas para la etapa primaria (Julián, Abarca-Sos, Zaragoza y Aibar, 2016; Méndez, Fernández-Río, Méndez-Giménez y Prieto Saborit, 2015; Muñoz, 2014).

Por ello, es objeto general de este trabajo realizar un análisis de los aspectos evaluativos en Educación Física comparando los 17 currículos autonómicos que emanan del reglamento estatal preceptuado en el Real Decreto 126/2014, de 28 de febrero, por el que se establece el currículo básico de la Educación Primaria. De forma más operativa, formulamos los siguientes objetivos:

1. Extraer patrones estadísticos descriptivos que exploren los currículos de EF en las variables analizadas.

2. Comparar aspectos cuantitativos y cualitativos de la evaluativos en EF entre los 17 currículos autonómicos en Educación Primaria.

\section{Método}

Para la comparación de los diferentes currículos autonómicos se utilizó el análisis documental como instrumento de obtención informativa. Para ello, se obtuvo la información de los 17 reglamentos autonómicos que desarrollan los aspectos evaluativos en EF. Todos los documentos curriculares circularon por tres expertos en la materia curricular. Tras la primera circulación se convinieron los aspectos comunes a categorizar y analizar en los 17 documentos curriculares.

Resultaron un total de 12 variables a comparar y analizar. Por un lado, variables cualitativas. Un total de 9 variables con la siguiente descripción: existencia de objetivos de área, criterios y estándares; relación entre criterios y estándares; tipo de secuenciación de los estándares; relación curricular de los estándares; y existencia de orientaciones metodológicas evaluativas al margen de los EA y CE. Por otro lado, como resultado del análisis de las variables cualitativas, resultaron tres indicadores cuantitativos: número de objetivos, número de criterios y números de estándares.

El procedimiento para la categorización fue a través de una hoja de control con las 12 variables al tiempo que se analizaban los 17 currículos autonómicos que desarrollan el RD 126/2014 por el que se establece el currículo básico. Todos los currículos autonómicos están publicados en los respectivos boletines oficiales de sus Comunidades Autónomas. Se encuentran en formato pdf y son de acceso libre y gratuito. Tras una primera categorización se procedió al análisis en el programa SPSS v. 17. Para las 9 variables cualitativas se analizaron las Frecuencias y Porcentajes y para las 3 cuantitativas se analizaron los estadísticos descriptivos.

\section{Resultados}

Un análisis exploratorio (Cuadro 1) nos muestra que sólo un 30\% (29,4\%) de las Comunidades incluyen objetivos propios de la asignatura de EF. Si bien en el caso de los CE hay unanimidad (el 100\% incluye criterios) el caso de los EA hay un 17\% que no los incluye. Para la variable ‘Tipo de secuenciación’ que se hace de EA y CE, mientras que la gran mayoría $(76,5 \%)$ secuencia por niveles educativos, un $17,6 \%$ los hace por ciclos y $5,9 \%$ por etapa. En la variable 'Relación Curricular' la mayoría de comunidades $(94,1 \%)$ 
relacionan $\mathrm{CE}$ y $\mathrm{EA}$ con los contenidos educativos, no ocurre así con elementos vertebradores como las competencias clave dónde sólo un 47,1\% (8 Comunidades) relacionan CE y EA con competencias clave. Por último, sólo un 17,6\% ha incluido orientaciones metodológicas que guíen a los maestros sobre cómo evaluar y utilizar los referentes evaluativos explicitados.

Cuadro 1. Frecuencias y porcentajes de las variables cualitativas que analizan los aspectos evaluativos del currículo de Educación Física en Primaria

\begin{tabular}{|c|c|c|c|c|}
\hline & FreCUENCIA & Porcentaje & $\begin{array}{l}\text { PORCENTAJE } \\
\text { VÁLIDO } \\
\end{array}$ & $\begin{array}{l}\text { PORCENTAJE } \\
\text { ACUMULADO } \\
\end{array}$ \\
\hline Existencia de Objetivos & 5,0 & 29,4 & 29,4 & 29,4 \\
\hline Existencia de criterios & 17,0 & 100,0 & 100,0 & 100,0 \\
\hline Existencia de estándares & 14,0 & 82,4 & 82,4 & 82,4 \\
\hline Relación criterios y estándares & 14,0 & 82,4 & 82,4 & 82,4 \\
\hline \multirow{2}{*}{$\begin{array}{l}\text { Tipo de } \\
\text { secuenciación }\end{array}$} & 13,0 & 76,5 & 76,5 & 76,5 \\
\hline & 3,0 & 17,6 & 17,6 & 94,1 \\
\hline secuenciacion Etapa & 1,0 & 5,9 & 5,9 & 100,0 \\
\hline \multirow{3}{*}{$\begin{array}{lc}\text { Relación } & \text { CC } \\
\text { curricular } & \text { CT } \\
\text { Orientaciones } & \text { evaluativas } \\
\end{array}$} & 8,0 & 47,1 & 47,1 & 47,1 \\
\hline & 16,0 & 94,1 & 94,1 & 94,1 \\
\hline & 3,0 & 17,6 & 17,6 & 17,6 \\
\hline
\end{tabular}

Fuente: Elaboración propia.

Sobre las variables anteriores, a nivel cuantitativo, el análisis del número de objetivos, criterios y estándares nos muestra diferencias sustanciales (Cuadro 2). Mientras que en el 'Número de objetivos' la baja media aritmética $(\mathrm{M}=2,59)$ y la alta desviación estándar $(3,97)$ se deben al alto número de comunidades que incluyen objetivos en su currículo, en el caso de los dos referentes evaluativos, existe un elevado promedio en el número de $\mathrm{CE}$ $(\mathrm{M}=61,53)$ y de $\mathrm{EA}(\mathrm{M}=200,24)$. En ambos, y pesar de la que mayoría de Comunidades los incluyen en su currículo, la heterogeneidad en el número es muy alta como muestran las desviaciones estándar ( $\mathrm{DE}=61,44$ y 144,69 respectivamente)

Cuadro 2. Estadísticos descriptivos variables cuantitativas que analizan los aspectos evaluativos del currículo de Educación Física en Primaria

\begin{tabular}{lrrrrc}
\hline & N & Mínimo & MÁximo & MEdia & $\begin{array}{l}\text { DESVIACIóN } \\
\text { ESTÁNDAR }\end{array}$ \\
\hline Número de objetivos & 17 & 0 & 10 & 2,59 & 3,97 \\
Número de criterios & 17 & 8 & 253 & 61,53 & 61,4 \\
Número de estándares & 17 & 0 & 481 & 200,24 & 144,69 \\
\hline
\end{tabular}

Fuente: Elaboración propia.

Otros de los objetivos de este trabajo era comparar aspectos cuantitativos y cualitativos de la evaluativos en EF entre los 17 currículos autonómicos en Educación Primaria (Cuadro 3). La codificación que comparaba los currículos de EF en las 17 comunidades ofrece características diversas en las diferentes variables. Sobre la 'Existencia de objetivos' sólo 5 comunidades de las 17 (Andalucía, Aragón, Baleares, Cataluña y País Vasco) optan por mantener los objetivos de la asignatura de EF que sí contemplaban los desarrollos curriculares previos a la LOMCE.

El resto de comunidades sólo contempla 'Criterios de Evaluación' y 'Estándares de Aprendizaje' como referentes evaluativos. En esas dos variables sí existe uniformidad cualitativa: todas contemplan CE y la mayoría EA (excepto Cataluña, País Murcia y País Vasco). Sí existe disparidad en cuanto al número de CE formulados. Mientras hay 
Comunidades con un número bajo de CE (8 de Canarias y País Vasco) otras lo elevan incluso por encima del número de EA que formulan otras comunidades. Es el caso de los CE de Castilla y León (91 CE), La Rioja (92 CE) o Aragón (95 CE). La diferencia numérica es mayor en los EA en donde encontramos comunidades que el número de EA ronda el medio millar (Cantabria 481 EA y Castilla-La Mancha 410) y otras como Andalucía, Canarias y Madrid que contemplan un número de reducido de estándares (4.1 y 44 respectivamente).

Cuadro 3. Comparación y codificación de las variables cuantitativas y cualitativas de los aspectos los aspectos evaluativos del currículo de Educación Física en Primaria para la asignatura de Educación Física

\begin{tabular}{|c|c|c|c|c|c|c|c|c|c|c|c|}
\hline & \multicolumn{2}{|c|}{ OA } & \multicolumn{2}{|c|}{$\mathrm{CE}$} & \multicolumn{2}{|c|}{ EA } & \multirow{2}{*}{$\begin{array}{c}\text { RELA- } \\
\text { CIÓN CE } \\
\text { Y EA }\end{array}$} & \multirow{2}{*}{$\begin{array}{l}\text { SECuENCIA } \\
\text { CIÓN } \\
\text { DE CE Y EA }\end{array}$} & \multicolumn{2}{|c|}{$\begin{array}{c}\text { RELACIÓN } \\
\text { CURRICULA } \\
\text { R }\end{array}$} & \multirow[t]{2}{*}{$\begin{array}{l}\text { OR. } \\
\text { EV }\end{array}$} \\
\hline & $\mathbf{E}$ & $\mathbf{N}^{\circ}$ & $\mathbf{E}$ & $\mathbf{N}^{\circ}$ & $\mathbf{E}$ & $\mathbf{N}^{\circ}$ & & & $\mathrm{CC}$ & CT & \\
\hline Andalucía & Sí & 7 & Sí & 38 & Sí & 42 & Sí & Ciclo & Sí & Sí & Sí \\
\hline Aragón & Sí & 10 & Sí & 85 & Sí & 208 & Sí & Nivel & Sí & Sí & No \\
\hline Asturias & No & No & Sí & 13 & Sí & 292 & Sí & Nivel & No & Sí & No \\
\hline Baleares & Sí & 10 & Sí & 39 & Sí & 106 & Sí & Ciclo & No & Sí & $\mathrm{No}$ \\
\hline Canarias & No & No & Sí & 8 & Sí & 44 & Sí & Nivel & Sí & Sí & No \\
\hline Cantabria & $\mathrm{No}$ & $\mathrm{No}$ & Sí & 253 & Sí & 481 & Sí & Nivel & Sí & Sí & $\mathrm{No}$ \\
\hline Castilla-LM & No & No & Sí & 104 & Sí & 410 & Sí & Nivel & No & Sí & No \\
\hline C. y León & $\mathrm{No}$ & No & Sí & 91 & Sí & 333 & Sí & Nivel & No & Sí & No \\
\hline Cataluña & Sí & 8 & Sí & 25 & No & No & No & Ciclo & $\mathrm{Si}$ & Sí & Sí \\
\hline Extrema-dura & No & No & Sí & 13 & Sí & 281 & Sí & Nivel & No & Sí & No \\
\hline Galicia & No & No & Sí & 83 & Sí & 261 & Sí & Nivel & Sí & Sí & No \\
\hline La Rioja & No & No & Sí & 92 & Sí & 316 & Sí & Nivel & Sí & Sí & No \\
\hline C. de Madrid & No & No & Sí & 13 & Sí & 44 & Sí & Nivel & No & Sí & No \\
\hline R. de Murcia & No & No & Sí & 13 & No & 203 & No & Nivel & No & Sí & Sí \\
\hline Navarra & No & No & Sí & 67 & Sí & 197 & Sí & Nivel & No & Sí & No \\
\hline País Vasco & Sí & 7 & Sí & 8 & No & $\mathrm{O}$ & No & Etapa & No & No & No \\
\hline C. Valenciana & No & No & Sí & 101 & Sí & 184 & Sí & Nivel & Sí & Sí & No \\
\hline
\end{tabular}

Notas: OA: Objetivos de Área; E: Existen: $\mathrm{N}^{\circ}$ : Número; EA: Estándar de Aprendizaje; CC: Competencias Clave; T: Contenidos: OR. EV: Orientaciones Evaluativas.

Fuente: Elaboración propia.

Sobre si los documentos curriculares presentan una relación explícita entre CE y EA con cuadros y/o tablas en donde los más concretos estén contenidos en los más generales existe uniformidad excepto en las 3 comunidades citadas que no contemplan EA.

Cuestión diferente es lo ocurrido entre el tipo de secuenciación de los EA. Existen tres casos diferentes: etapa, ciclo o nivel. Mientras País Vasco es la única comunidad que los contempla por etapa, 3 comunidades (Andalucía, Cataluña y Baleares) que aún conservan la estructura de ciclo secuencian sus referentes evaluativos (CE y/o EA) por ciclo y el resto de Comunidades los organiza por nivel.

Sobre si los currículos autonómicos incluían CE y/o EA relacionándolos con otros elementos curriculares se tuvieron los dos más frecuentes: Competencias clave y contenidos. En la variable 'Relación CE y EA' se comprueba que sólo 9 Comunidades (Andalucía, Cantabria, Canarias, Cataluña, Galicia, LA Rioja, Comunidad Valenciana) relacionan sus referentes evaluativos con las competencias clave. Cuestión diferente ocurre en la variable 'Relación CE y EA' cuando hablamos de contenidos pues todas las Comunidades excepto País Vasco relacionan CE y/o EA con los contenidos de enseñanza. 
Por último, la 'OR. EV' tenía como pretensión indagar sobre si al margen de la CE y EA las Comunidades incluían orientaciones metodológicas para la evaluación. En este sentido, sólo Andalucía, Cataluña y Región de Murcia incluyen orientaciones sobre evaluación.

\section{Discusión}

Una primera interpretación de los resultados nos muestra que nos hallamos ante el estadio curricular en España que pone fin a los objetivos de área ya que sólo 5 de las 17 Comunidades los mantienen. Esta situación no puede entenderse de otra forma que como el acotamiento definitivo del currículo. Los objetivos de área eran formulados en términos generales de ahí su bajo número tanto en los desarrollos curriculares previos a la LOMCE como en los descritos en el presente artículos (Andalucía 7; Aragón, 10; Baleares; 10; Cataluña, 8; País Vasco, 7). Esa ausencia de constricción en su formulación permitía que los docentes y alumnado fabricaran un currículo en base a sus necesidades. Sobre este aspecto arroja luz la sentencia de Stenhouse (1987) al formular que el currículo "debe ser puesto a prueba por estudiantes y profesores" (currículo abierto) "y no estudiantes y profesores" puesto a pruebas por el currículo (currículo cerrado). En este sentido un mecanismo para un currículo como Proyecto y Proceso es que docentes y alumnado formularan sus criterios de evaluación contextualizados en situaciones de aprendizaje propias, e incardinándose en el primer nivel de concreción en base a objetivos de área y criterios de evaluación regulados por los respectivos reglamentos.

No obstante, la prueba de que el exterminio de los objetivos de área evoluciona hacia un cerrazón del currículo es que el otro elemento curricular de este trabajo CE, aún no siendo un elemento nuevo (ya existía en la etapa pre-LOMCE), la novedad es el desorbitado promedio $(\mathrm{M}=61,53)$ si los comparamos con el reducido número de criterios del desarrollo reglamentario previo a la LOMCE. En la actualidad nos encontramos picos de CE como 253 en Cantabria, 104 en Castilla y León o 101 en la Comunidad Valenciana. Sobre este grado de operativización y tecnificación de los aprendizajes llama poderosamente la atención que la retirada de autonomía al docente y vuelta al rol de técnico aplicador suceda en los CE, es decir, antes incluso de los EA que son "especificaciones de los criterios de evaluación”. Valga como ejemplo de la estandarización de los aprendizajes que hace cada comunidad el caso de autonomías que con un número de CE superior al de los EA de otras, siendo estos últimos concreciones de los primeros. No ocurre esto tan sólo con las Comunidades con las frecuencias más altas de $\mathrm{CE}$, sino en algunas cuya frecuencia (Navarra, $67 \mathrm{CE}$ ) se encuentra en el promedio español $(\mathrm{M}=61,53)$ y cuyo número de $\mathrm{CE}$ es incluso superior al número de EA de otras (Andalucía, 42, Canarias 44 o Madrid 44).

Como era de esperar, el promedio de EA es más chirriante $(\mathrm{M}=202,24)$ que el de los $\mathrm{CE}$, sobre todos si aterrizamos en las necesidades del aula y estamos ante el supuesto surrealista que el número de EA a evaluar (o medir) por los maestros y maestras de EF es superior a las sesiones que tienen durante un curso académico. Este supuesto (en realidad no es una hipótesis, sino la receta de los 17 legisladores a sus docentes desde hace varios años) se sale de la lógica aún más cuando con la aritmética más simple se comprueba que hay comunidades tienen tantos EA como número de sesiones de EF (Asturias 292 EA; Cantabria 481; Castilla-La Mancha, 410; Extremadura 281; La Rioja, 216). Llegados a este punto, no estamos ante una única discusión cuantitativa ni tampoco sobre un debate para 
consensuar un número idóneo de estándares. Es una cuestión pedagógica más simple: en cada lugar se aprende de diferente forma (Pozo, 2016).

Sin embargo, el Real Decreto 126/2014 del que emanan los currículos autonómicos marcó unas directrices evaluativas de estandarización para todas las asignaturas. Si bien se habla someramente del carácter "global" de la evaluación, no se hace mención ni siquiera de pasada (cosa que sí hacen algunos reglamentos autonómicos posteriormente) al carácter formativo de la evaluación ni, por supuesto, a la participación del alumnado en la misma. En este sentido, cabe resaltar la incoherencia de que difícilmente una evaluación puede ser global si se constriñe tanto (hasta casi medio millar de EA en algunas Comunidades). Esta situación desemboca en una operativización tal que nos retrotrae a la tendencia de los otroras objetivos operativos dividían las dimensiones del ser humano (cognitivo, motriz y afectivo cada uno formulados en EA diferentes) a sabiendas de que en cada respuesta de nuestro alumnado se actúa como un todo.

Con un hartazgo tal de operativización en los EA cabe preguntarse, ¿qué estamos midiendo? La respuesta a esta pregunta la ofrece textualmente Real Decreto 126/2014 cuando se refiere a sólo aquello "observable", "medible" y con carácter de "rendimiento". Con los tres atributos anteriores se plantea la siguiente cuestión ¿̇se valoran cualidades tan en boga y cuyo enterramiento se les atribuye a la escuela como la creatividad, la empatía o la persistencia? La respuesta a esta pregunta la ofrece Biesta (2014) con otro interrogante ¿Medimos lo que valoramos o valoramos lo que medimos? Dicho de otra forma, los currículos han puesto en valor sólo aquello que puede operativizar y cuantificar ("observable", "medible" y "de rendimiento") como si nuestro alumnado formara parte de una cadena de producción industrial o fuera un elemento más de una cuenta de resultado empresarial. En el caso de los currículos de EF los EA aquello más fácil de medir se ciñe exclusivamente a la dimensión motriz, renunciando al carácter global y, por, ende educativo de la evaluación (Ejemplos de EA. Cantabria: 'Conoce para qué sirve cada uno de los cinco sentidos'; Castilla-La Mancha: 'Es capaz de realizar una actividad de nivel moderado-vigoroso entre 6 y 8 minutos'; Navarra: 'Se coloca y se desplaza con respecto a los objetos orientados en diferentes posiciones'). Es fácilmente reconocible tras la lectura de los 17 currículos que aquellos currículos con un mayor número de EA tienden hacia una formulación más conductista y motriz. En los casos con menor frecuencia de EA, la formulación es más global e integra las diferentes dimensiones del ser humano. Es necesario señalar que, aunque tarde y a modo de declaración de intenciones, es decir, sin el grado de prescripción del Real Decreto 126/2014, hay un leve acercamiento al carácter formativo de la evaluación en el artículo 7 de un reglamento anexo (Orden ECD/65/2015, de 21 de enero). No obstante, a nuestro entender, y tras todo lo expuesto en este trabajo, este acercamiento es insuficiente y carece del liderazgo pedagógico y del reconocimiento docente que sí tienen los reglamentos curriculares autonómicos.

Tras la fotografía evaluativa vislumbrada, es razonable que la mayoría $(76,5 \%)$ de currículos hayan secuenciado $\mathrm{CE}$ y/o EA por niveles educativos en lugar de los desaparecidos ciclos $(17,6 \%)$. En la misma línea de falta de globalidad evaluativa era de esperar que sólo menos de la mitad de currículos $(47,1 \%)$ relacionen sus referentes evaluativos con las manidas Competencias Clave. En cualquiera de los casos, y valorando que muchos profesionales de las aulas pudieran agradecer y facilitarle su docencia la existencia de guías y estándares de evaluación (en algunas Comunidades con aplicaciones informáticas incluidas), los currículos autonómicos tenían la oportunidad de, formulando 
referentes evaluativos tan concretos, facilitar estrategias de evaluación formativa y compartida (López-Pastor y Pérez-Pueyo, 2017). Los legisladores pudieran haber prescrito - ahora también, cómo no-, mecanismos de coevaluación o autoevaluación entre alumnado de una forma tan concretada como se ha hecho con EA y CE. En definitiva, concretar también una evaluación que pusiera el foco en el aprendizaje no sólo en la medición de conductas. Sin embargo, llama la atención que sólo 3 de los 17 currículos (Andalucía, Cataluña y Murcia) incluyen en sus documentos curriculares orientaciones de este tipo. Estaríamos sentenciando objetivamente (y no interpretando) si afirmamos que el 82,4\% de los currículos de España no orienta a su profesorado sobre cómo realizar una evaluación educativa, aunque sí detalle cómo deben medir los aprendizajes. Cabe señalar que sin bien muchas comunidades poseen reglamentos específicos de evaluación, la mayor parte de ellos se dedica al carácter administrativo de la evaluación (documentales oficiales, plazos, calificaciones...) y que las pocas o inexistentes menciones educativas de la evaluación son someras y de poco calado ante el profesorado.

\section{Conclusiones}

Tras lo expuesto anteriormente podemos concluir que la LOMCE y su concreción en los currículos autonómicos han supuesto una involución pedagógica en materia de evaluación. En el caso concreto de la asignatura de EF la regresión tiene una tendencia hacia la medición, que no evaluación, preponderante de aspectos motrices (Méndez, FernándezRío, Méndez Giménez y Prieto Saborit, 2015). Esta tendencia exclusivamente operativizadora ha retrotraído, además, la evaluación en Educación Física a la antigua división de las dimensiones en cognitiva, motriz y afectivo-social focalizando lo motriz y separándolo, en ocasiones, en compartimentos estancos de las otras dimensiones.

Futuros trabajos pudieran abordar el estudio estadístico y taxonómico del más del millar de CE y los tres millares y medio de EA. Así mismo, y dado que la tendencia de medición pone en entredicho el derecho del alumnado a ser educado y no adiestrado (Teach to test), existen mecanismos de autonomía pedagógica que pueden poner a salvo la evaluación educativa. Entre otras propuestas, entendemos que el tercer nivel de concreción debe incentivar la construcción de objetivos (aún sin prohibir) y criterios didácticos que evolucionen hacia un modelo de docente investigador de su propia práctica. Por otra parte, las Administrativas Educativas autonómicas tienen la posibilidad de legislar orientaciones de evaluación educativas que dibujen una evaluación más democrática y adaptada a las necesidades del alumnado y a un currículo no industrializado más propio del siglo XXI.

\section{Referencias}

Alcaraz-Salariche, N. (2015). Evaluación versus calificación. Revista Aula de Encuentro 17(2), 209236.

Biesta, G. (2014). ¿Medir lo que valoramos o valorar lo que medimos? Globalización, responsabilidad y la noción de propósito de la educación. Pensamiento Educativo. Revista de Investigación Educacional Latinoamericana. 51(1), 46-57

Cronbach, L. J. (1975). Beyond the two disciplines of scientific psychology. American Psychologist, 30(2), 116-127. https://doi.org/10.1037/ho076829 
Decreto 97/2015, de 3 de marzo, por el que se establece la ordenación y el currículo de la Educación Primaria en la Comunidad Autónoma de Andalucía. (BOJA nº 50, 13 de marzo de 2015)

Decreto 82/2014, de 28 de agosto, por el que se regula la ordenación y establece el currículo de la Educación Primaria en el Principado de Asturias (BOPA, nº 202, 30 de agosto de 2014).

Decreto 32/2014 de 18 de julio, por el que se establece el currículo de la educación primaria en las Illes Balears (BOIB nº 97, 19 de julio de 2014).

Decreto 89/2014, de 1 de agosto, por el que se establece la ordenación y el currículo de la Educación Primaria en la Comunidad Autónoma de Canarias (BOC n ${ }^{\circ}$ 156, 13 de agosto de 2014).

Decreto 27/2014 de 5 de junio que establece el currículo de Educación Primaria en la comunidad autónoma de Cantabria (BOC nº 29, 13 de junio de 2014).

Decreto 54/2014, de 10/07/2014, por el que se establece el currículo de la Educación Primaria en la Comunidad Autónoma de Castilla-La Mancha (DOCM nº 132, 11 de julio de 2014).

Decret 119/2015, de 23 de juny, d'ordenació dels ensenyaments de l'educació primària (DOGC nº 6900, de 26 de junio de 2015 )

Decreto 103/2014, de 10 de junio, por el que se establece el currículo de Educación Primaria para la Comunidad Autónoma de Extremadura (DOE nº 114, 16 de junio de 2014).

Decreto24/2014, de13dejunio, por el que se establece el currículo de la Educación Primaria en la Comunidad Autónoma de La Rioja (BOR nº 74, 16 de junio de 2014).

Decreto 89/2014, de 24 de julio, del Consejo de Gobierno, por el que se establece para la Comunidad de Madrid el Currículo de la Educación Primaria (BOCM nº 175, 25 de julio de 2014).

Decreto 198/2014, de 5 de septiembre, por el que se establece el Currículo de la Educación Primaria en la Comunidad Autónoma de la Región de Murcia (BORM nº 206, 6 de septiembre de 2014).

Decreto Foral 60/2014 de 16 de junio por el que se establece el currículo de Educación Primaria de la comunidad Foral de Navarra (BON n ${ }^{\circ}$ 174, 5 de septiembre de 2014).

Decreto 236/2015, de 22 de diciembre, por el que se establece el currículo de Educación Básica y se implanta en la Comunidad Autónoma del País Vasco (BOPV nº 9, de 15 de enero de 2016)

Decreto 108/2014, de 4 de julio, del Consell, por el que establece el currículo y desarrolla la ordenación general de la educación primaria en la Comunitat Valenciana (DOCV $n^{\circ} 7311$, de 7 de julio de 2014).

Díez-Gutiérrez, E. (2018). Neoliberalismo educativo. Barcelona: Octaedro.

Hopkins, D. (1989). Investigación en el aula. Barcelona: PPU.

Julián, J.A., Abarca-Sos, A., Zaragoza, J. y Aibar, J. (2016). Análisis crítico de la propuesta del currículum básico de la LOMCE para la asignatura de Educación Física. Acciones derivadas en la Comunidad Autónoma de Aragón y propuestas de futuro. 2016, Retos, 29, 173-181.

Lamb, P., Lane, K. y Aldou, D. (2013). Enhancing the Spaces of Reflection: A Buddy Peer-Review Process within Physical Education Initial Teacher Education. European Physical Education Review 19(1), 21-38. https://doi.org/10.1177/1356336X12457293

López-Pastor, V. M. (1999). Prácticas de evaluación en educación física: Estudio de casos en Primaria, Secundaria y formación del profesorado. Tesis doctoral publicada. Valladolid: Secretariado de Publicaciones de la Universidad de Valladolid. 
López-Pastor, V. M. (2004). Evaluación, Calificación, Credencialismo y formación inicial del Profesorado: Efectos y patologías generadas en la enseñanza universitaria. Revista Interuniversitaria de Formación del Profesorado (51), 22 1-232.

López-Pastor, V.M. y Pérez-Pueyo, A. (2017) Evaluación formativa y compartida en educación: experiencias de éxito en todas las etapas educativas. León: Secretariado de Publicaciones de la Universidad de León.

López-Pastor, V. M., Barba, J. J., Monjas, R., Manrique, J. C., Heras, C., González, M. y Gómez, J. M. (2007). Trece años de evaluación compartida en educación física. Revista Internacional de Ciencias de la Actividad Física y el Deporte, 7(26), 69-86

Lorente-Catalán, E. y Kirk. D. (2014). Making the Case for Democratic Assessment Practices within a Critical Pedagogy of Physical Education Teacher Education. European Physical Education Review 2O(1), 104-119. https://doi.org/10.1177/1356336X13496004

Méndez, D., Fernández-Río, J., Méndez-Giménez, A. y Prieto, J. A. (2015). Análisis de los currículos autonómicos LOMCE de Educación Física en Educación Primaria. Retos, 28, 1520 .

Muñoz, J. C. (2014). El currículo del área de educación física de primaria en la lomce. Análisis del Real Decreto 126/2014. EmásF: Revista Digital de Educación Física, 27, 24-30.

Ní Chróinín, D. y C. Cosgrave. (2013). Implementing Formative Assessment in Primary Physical Education: Teacher Perspectives and Experiences. Physical Education and Sport Pedagogy, 18(2), 219-233. https://doi.org/10.1080/17408989.2012.666787

Orden de 16 de junio de 2014, de la Consejera de Educación, Universidad, Cultura y Deporte, por la que se aprueba el currículo de la Educación Primaria y se autoriza su aplicación en los centros docentes de la Comunidad Autónoma de Aragón (BOA nº 119, 20 de junio de 2014).

Orden EDU/519/2014, de 17 de junio, por la que se establece el currículo y se regula la implantación, evaluación y desarrollo de la educación primaria en la Comunidad de Castilla y León (BOCL n ${ }^{\circ}$ 117,20 de junio de 2014).

Orden de 23 de julio de 2014 por la que se regula la implantación para el curso 2014/15 de los cursos primero, tercero y quinto de educación primaria en la Comunidad Autónoma de Galicia, según el calendario de aplicación de la Ley orgánica 8/2013, para la mejora de la calidad educativa (DOG, $\mathrm{n}^{\circ} 154,14$ de agosto de 2014).

Otero, F. M. (2013). Información y cesión de la responsabilidad en la evaluación de los deportes de invasión en Primaria. EmásF: Revista Digital de Educación Física, 22, 56-71.

Otero, F. M., Calvo, A. y González-Jurado, J. A. (2014). Analysis of the Assessment of Invasion Sports in Elementary School. Cultura, Ciencia y Deporte, 9(26), 139-153.

Pérez, A., Heras, B. y Herrán, I. (2008). Evaluación formativa en la Educación Secundaria Obligatoria. Su aplicación a una unidad didáctica de deportes colectivos en el marco de un estilo actitudinal. Revista Española de Educación Física y Deportes, 9, 14-25.

Pozo, I. (2016). Aprender en tiempos revueltos. Madrid: Alianza.

Real Decreto 126/2014, de 28 de febrero, por el que se establece el currículo básico de la Educación Primaria (BOE núm 52, 1/3/2014).

Santos, M. A. (2006). Una flecha en la diana: La Evaluación como Aprendizaje. Madrid: Narcea.

Stenhouse, L. (1987). La investigación como base de la enseñanza. Madrid: Morata. 
Vera, J. A., González, R. y Moreno, J.A. (2009). Relationships between the transfer of responsibility in assessment and the perception of equality in physical education classes. Cultura, Ciencia y

Deporte, 4(10), 25-31.

\section{Cv de los autores}

\section{Fernando Manuel Otero-Saborido}

En la actualidad ejerce como profesor a tiempo completo en la Universidad pública Pablo de Olavide de Sevilla. Anteriormente, funcionario de carrera del Cuerpo de Maestros y del Cuerpo de Profesores de Secundaria en Andalucía, ambos en la especialidad de Educación Física. Formación académica en todas las titulaciones oficiales universitarias en Educación Física: Doctor; Máster Oficial en Educación Físico-Deportiva; Licenciado en Ciencias de la Actividad Física y del Deporte; Diplomado en Magisterio (Ed. Física). A lo largo de 25 artículos de revistas, su línea de investigación se centra en procesos de evaluación y metodología de enseñanza Educación Física y el Deporte así como aspectos relacionados con el rendimiento físico deportivo en fútbol. ORCID ID: 0000-0002-7016-2414. Email: fmotero@upo.es

\section{Francisco Javier Vázquez-Ramos}

Doctor por la Universidad de Sevilla, licenciado en Ciencias de la Actividad Física y el Deporte (Universidad de Granada), diplomado en Ciencias de la Educación especialidad de Educación Física (Universidad de Sevilla, CEU San Pablo) y entrenador nacional de Voleibol. Actualmente es maestro de Educación Física en la Consejería de Andalucía y componentes del grupo de investigación: Deporte y Sociedad: la Actividad FísicoDeportiva desde una Perspectiva Multidisciplinar (Universidad de Sevilla). Ha creado la plataforma digital de formación "El Valor de la Educación Física". En el año 2015 obtuvo el VI Premio a la Mejor Práctica Profesional de Carácter Innovador 2015, otorgado por el Ilustre Colegio Oficial de Licenciados en Ciencias de la Actividad Física y el Deporte de Andalucía. ORCID ID: 0000-0001-5646-2018. Email: kiscoarahal@gmail.com 